\title{
Independent factorization of the last zero arcsine law for Bessel processes with drift*
}

\author{
Hugo Panzo ${ }^{\dagger}$
}

\begin{abstract}
We show that the last zero before time $t$ of a recurrent Bessel process with drift starting at 0 has the same distribution as the product of a right-censored exponential random variable and an independent beta random variable. This extends a recent result of Schulte-Geers and Stadje [19] from Brownian motion with drift to recurrent Bessel processes with drift. We give two proofs, one of which is intuitive, direct, and avoids heavy computations. For this we develop a novel additive decomposition for the square of a Bessel process with drift that may be of independent interest.
\end{abstract}

Keywords: Bessel process with drift; Bessel bridge; arcsine law; time inversion property; additivity property; last exit time.

MSC2020 subject classifications: Primary 60G17; 60J60, Secondary 60J65.

Submitted to ECP on December 4, 2020, final version accepted on May 27, 2021.

Supersedes arXiv: 2010.00579v2.

\section{Introduction}

Let $X=\left(X_{t}: t \geq 0\right)$ denote the coordinate process on the canonical space of continuous functions from $[0, \infty)$ to $\mathbb{R}$ and let $\mathbb{W}_{x}$ denote the law under which $X$ is Brownian motion starting at $x \in \mathbb{R}$. We write $g_{t}$ for the last zero of $X$ before time $t$. More precisely, $g_{t}=\sup \left\{s \leq t: X_{s}=0\right\}$ with the usual convention of $\sup \{\emptyset\}=-\infty$. The well-known arcsine law for $g_{t}$ is due to Lévy [8, § 7.7] and states that

$$
g_{t} \text { under } \mathrm{W}_{0} \stackrel{\mathcal{L}}{=} t A_{\frac{1}{2}}
$$

where we used $\stackrel{\mathcal{L}}{=}$ to indicate equality in law and $A_{\frac{1}{2}}$ is a $\operatorname{Beta}\left(\frac{1}{2}, \frac{1}{2}\right)$ random variable with density

$$
P\left(A_{\frac{1}{2}} \in \mathrm{d} x\right)=\frac{1}{\pi \sqrt{x(1-x)}} \mathbb{1}_{(0,1)}(x) \mathrm{d} x .
$$

There have been many extensions of (1.1) to processes besides 1-dimensional Brownian motion and the reader is directed to Chapter 8 of [10] and Section 2.5 of [12] as well as references therein for some examples. One such generalization that plays an important role in this paper is due to Lamperti [6, Theorem 5.1] and identifies the distribution of $g_{t}$ when the underlying Brownian motion is replaced by a recurrent Bessel

*Supported at the Technion by a Zuckerman Fellowship.

${ }^{\dagger}$ Technion - Israel Institute of Technology, Haifa 32000, Israel. E-mail: panzo@campus .technion.ac.il 
process of dimension $\delta \in(0,2)$. We use $\mathbb{P}_{x}^{\delta}$ to denote the law of this Bessel processes when it starts at $x \geq 0$. Lamperti showed that

$$
g_{t} \text { under } \mathbb{P}_{0}^{\delta} \stackrel{\mathcal{L}}{=} t A_{\alpha}
$$

where $\alpha=1-\frac{\delta}{2} \in(0,1)$ and $A_{\alpha}$ is a $\operatorname{Beta}(\alpha, 1-\alpha)$ random variable with density

$$
P\left(A_{\alpha} \in \mathrm{d} x\right)=\frac{x^{\alpha-1}(1-x)^{-\alpha}}{\Gamma(\alpha) \Gamma(1-\alpha)} \mathbb{1}_{(0,1)}(x) \mathrm{d} x .
$$

Lévy's last zero arcsine law (1.1) can be seen as a special case of (1.2) since the law of $|X|$ under $\mathbb{W}_{0}$ is the same as the law of $X$ under $\mathbb{P}_{0}^{1}$.

Another more recent generalization that is central to our results is that of characterizing the law of $g_{t}$ when a constant drift $\mu \in \mathbb{R}$ is added to the underlying Brownian motion. We use $\mathbb{W}_{x}^{\mu}$ to denote the law of Brownian motion with drift $\mu$ when it starts at $x \in \mathbb{R}$. Using a random walk approximation argument, Schulte-Geers and Stadje [19, Theorem 2.1] show that $g_{t}$ under $\mathbb{W}_{0}^{\mu}$ has the independent factorization

$$
g_{t} \text { under } \mathrm{W}_{0}^{\mu} \stackrel{\mathcal{L}}{=} \min \left\{t, E_{\mu}\right\} A_{\frac{1}{2}}
$$

where $E_{\mu}$ is an $\operatorname{Exp}\left(\frac{1}{2} \mu^{2}\right)$ random variable independent of $A_{\frac{1}{2}}$ and with density

$$
P\left(E_{\mu} \in \mathrm{d} t\right)=\frac{1}{2} \mu^{2} e^{-\frac{1}{2} \mu^{2} t} \mathbb{1}_{[0, \infty)}(t) \mathrm{d} t .
$$

Despite the elegant and elementary nature of (1.3), it seems to have escaped notice until [19]; see also [4, Remark 2.1]. The present author learned of this result from a MathOverflow answer [3] posted by the first author of [19]. Another attractive feature of (1.3) is that it allows us to easily recover Lévy's last zero arcsine law (1.1) since $\min \left\{t, E_{\mu}\right\}$ degenerates to $t$ as $\mu \rightarrow 0$. Moreover, since the last exit time from 0 is almost surely finite when $\mu \neq 0$, we can also recover the law of $g_{\infty}$ in this case. Letting $t \rightarrow \infty$ in (1.3) shows that $g_{\infty}$ under $\mathbb{W}_{0}^{\mu}$ is distributed like $E_{\mu} A_{\frac{1}{2}}$. After recalling the beta-gamma algebra [17, Chapter 0.6], it follows that $g_{\infty}$ under $\mathbb{W}_{0}^{\mu}$ is a $\operatorname{Gamma}\left(\frac{1}{2}, \frac{1}{2} \mu^{2}\right)$ random variable with density

$$
\mathbb{W}_{0}^{\mu}\left(g_{\infty} \in \mathrm{d} t\right)=\frac{|\mu|}{\sqrt{2 \pi t}} e^{-\frac{1}{2} \mu^{2} t} \mathbb{1}_{(0, \infty)}(t) \mathrm{d} t .
$$

While verifying (1.3) directly using a Girsanov measure change argument is a straightforward matter, tedious calculations are required as can be witnessed in Section 2 of [4] where this is carried out in detail. Another derivation which uses a formula from Borodin and Salminen's handbook [2] also requires significant computations; see Section 4.2. Indeed, in [19, Remark 2.3], the authors appeal for a "purely Brownian" explanation of the independent factorization (1.3). This leads us to the main contributions of this paper:

1. Extending (1.3) to Bessel processes of dimension $\delta \in(0,2)$ with positive drift (in the sense of Watanabe [20]), thereby unifying the last zero arcsine laws for Brownian motion (1.1) and recurrent Bessel processes (1.2) under the independent factorization framework of (1.3) when drift is present.

2. Giving a "purely Bessel" explanation for the independent factorization (1.3) and the aforementioned extension to Bessel processes with drift that is intuitive, direct, and avoids heavy computation. For this we develop an additive decomposition for the square of a Bessel process with drift which appears to be new and may be of independent interest. 
The remainder of the paper is organized as follows. In Section 2 we recall the definition of Bessel processes with drift and state our main theorems. In Section 3, we review several relevant properties of Bessel processes and Bessel bridges and prove some preliminary results. Finally, the main theorems are proved in Section 4.

\section{Main results}

\subsection{Bessel processes with drift}

Before stating our main results, we first recall the Bessel processes with drift introduced by Watanabe [20]. These comprise a two-parameter family of diffusions on $[0, \infty)$ that are indexed by their dimension $\delta>0$ and drift $\mu \geq 0$. Let $I_{\nu}$ denote the modified Bessel function of the first kind and for $\mu>0$, define the function $h_{\delta, \mu}:[0, \infty) \rightarrow[1, \infty)$ by

$$
h_{\delta, \mu}(x)= \begin{cases}1 & x=0 \\ \left(\frac{2}{\mu x}\right)^{\frac{\delta}{2}-1} \Gamma\left(\frac{\delta}{2}\right) I_{\frac{\delta}{2}-1}(\mu x) & x>0 .\end{cases}
$$

When $\mu>0$, the Bessel processes with drift are determined by the generator

$$
L^{\delta, \mu}=\frac{1}{2} \frac{\mathrm{d}^{2}}{\mathrm{~d} x^{2}}+\left(\frac{\delta-1}{2 x}+\frac{h_{\delta, \mu}^{\prime}(x)}{h_{\delta, \mu}(x)}\right) \frac{\mathrm{d}}{\mathrm{d} x}
$$

with 0 being a regular boundary with instantaneous reflection if $0<\delta<2$ or an entrance boundary if $\delta \geq 2$. When $\mu=0$, these processes coincide with the usual Bessel processes without drift having the same dimension. Accordingly, we use $\mathbb{P}_{x}^{\delta, \mu}$ to denote the law of a Bessel process with dimension $\delta$ and drift $\mu$ that starts from $x \geq 0$. By writing $\mathbb{P}_{x}^{\delta}$ instead of $\mathbb{P}_{x}^{\delta, 0}$, this notation subsumes that of the Bessel processes without drift from Section 1. We will also work with squared Bessel processes, both with and without drift, and will use $\mathbb{Q}_{x}^{\delta, \mu}$ and $\mathbb{Q}_{x}^{\delta}$, respectively, to denote the law of these processes. More precisely,

$$
\begin{gathered}
\left(X_{t}: t \geq 0 ; \mathbb{Q}_{x}^{\delta, \mu}\right) \stackrel{\mathcal{L}}{=}\left(X_{t}^{2}: t \geq 0 ; \mathbb{P}_{\sqrt{x}}^{\delta, \mu}\right) \\
\left(X_{t}: t \geq 0 ; \mathbb{Q}_{x}^{\delta}\right) \stackrel{\mathcal{L}}{=}\left(X_{t}^{2}: t \geq 0 ; \mathbb{P}_{\sqrt{x}}^{\delta}\right) .
\end{gathered}
$$

The appearance of the logarithmic derivative of $h_{\delta, \mu}$ in the first-order term of the generator (2.1) along with the fact that $L^{\delta, 0} h_{\delta, \mu}=\frac{1}{2} \mu^{2} h_{\delta, \mu}$ implies that a Bessel process of dimension $\delta$ with drift $\mu$ is simply a Bessel process of the same dimension without drift killed at rate $\frac{1}{2} \mu^{2}$ and then $h$-transformed by $h_{\delta, \mu}$. In particular, for any fixed $t \geq 0$, this gives the absolute continuity relation

$$
\left.\mathrm{d} \mathbb{P}_{x}^{\delta, \mu}\right|_{\mathcal{F}_{t}}=\left.e^{-\frac{1}{2} \mu^{2} t} \frac{h_{\delta, \mu}\left(X_{t}\right)}{h_{\delta, \mu}(x)} \mathrm{dP}_{x}^{\delta}\right|_{\mathcal{F}_{t}}
$$

where we used $\left(\mathcal{F}_{t}: t \geq 0\right)$ to denote the canonical filtration. Refer to [11, Section 4.1] for the requisite theory on $h$-transforms of diffusion processes.

As remarked upon in [14], the name "Bessel process with drift" is appropriate since for $\delta \in \mathbb{N}$, the law of $X$ under $\mathbb{P}_{0}^{\delta, \mu}$ is the same as that of the modulus of Brownian motion in $\mathbb{R}^{\delta}$ starting at 0 with a constant drift vector of magnitude $\mu$; see [18, Theorem 3]. Moreover, by the corollary to [20, Theorem 2.1], we have for any $\delta>0$ and $x, \mu \geq 0$

$$
\mathbb{P}_{x}^{\delta, \mu}\left(\lim _{t \rightarrow \infty} \frac{X_{t}}{t}=\mu\right)=1 .
$$

Interest in these processes is motivated in part by their being, up to a scale factor, the only regular and conservative diffusions on $[0, \infty)$ that satisfy the time inversion 
property [20, 7]; see Section 3.1 for more on this property. Additionally, when $\delta=3$ they make an appearance in Williams' path decomposition for Brownian motion with drift $[21,16]$. When $\delta=3$ and $\mu=1$, they also coincide with the hyperbolic Bessel process of dimension 3; see [5, 1]. We note that they are distinct from the Bessel processes with constant "naive drift" which arise in studies of bird navigation and queueing theory; see $[22,9]$ and references therein.

\subsection{Main theorems}

Our first result is an additive decomposition of $\left(X_{t}: t \geq 0\right)$ under $\mathbb{Q}_{0}^{\delta, \mu}$ into two independent processes that start at 0 : one being a squared Bessel process of dimension $\delta$ without drift and the other being a squared Bessel process of dimension 4 with drift $\mu$ which waits for an independent $\operatorname{Exp}\left(\frac{1}{2} \mu^{2}\right)$ time before starting. Such a decomposition was alluded to by Pitman and Yor in their Remark 5.8.iii of [15] but as far as we know, no explicit statement has ever appeared in the literature. A random waiting time before starting has featured in a similar additive decomposition for a squared Bessel process of dimension 2 without drift that appears in Section 3.5.1 of [10]. We adopt Mansuy and Yor's notation which makes use of the positive part $x^{+}:=\max \{x, 0\}$.

Theorem 2.1. Suppose $\delta, \mu>0$ and let $E_{\mu}$ be an independent $\operatorname{Exp}\left(\frac{1}{2} \mu^{2}\right)$ random variable. Then we have

$$
\left(X_{t}: t \geq 0 ; \mathbb{Q}_{0}^{\delta, \mu}\right) \stackrel{\mathcal{L}}{=}\left(X_{t}+X_{\left(t-E_{\mu}\right)^{+}}^{\prime}: t \geq 0 ; \mathbb{Q}_{0}^{\delta}(X) \times \mathbb{Q}_{0}^{4, \mu}\left(X^{\prime}\right)\right)
$$

where the product law notation $\mathbb{Q}_{0}^{\delta}(X) \times \mathbb{Q}_{0}^{4, \mu}\left(X^{\prime}\right)$ indicates that $X$ and $X^{\prime}$ are independent with laws $\mathbb{Q}_{0}^{\delta}$ and $\mathbb{Q}_{0}^{4, \mu}$, respectively.

Remark 2.2. The piecewise nature of the second summand distinguishes the additivity exhibited in Theorem 2.1 from the usual kind described in Section 3.4. A similarly exotic form of additivity for squared Bessel processes without drift and with possibly negative dimensions can be found in [13, Proposition 1.1].

Theorem 2.1 allows us to give a quick and intuitive proof of the independent factorization of the last zero arcsine law (1.3) and its generalization to Bessel processes with drift which we state below as Theorem 2.3.

Theorem 2.3. Let $g_{t}=\sup \left\{s \leq t: X_{s}=0\right\}$ be the last zero before time $t$ of a Bessel process with dimension $\delta \in(0,2)$ and drift $\mu>0$ starting at 0 . Put $\alpha=1-\frac{\delta}{2}$ and let $A_{\alpha}$ and $E_{\mu}$ be independent $\operatorname{Beta}(\alpha, 1-\alpha)$ and $\operatorname{Exp}\left(\frac{1}{2} \mu^{2}\right)$ random variables, respectively. Then we have the independent factorization

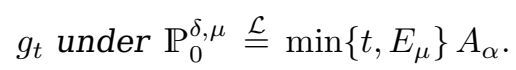

Remark 2.4. As mentioned before in Section $2.1,|X|$ under $\mathbb{W}_{0}^{\mu}$ has the same law as $X$ under $\mathbb{P}_{0}^{1,|\mu|}$ so it follows that (1.3) is a special case of Theorem 2.3.

Remark 2.5. We can recover Lamperti's arcsine law (1.2) by letting $\mu \rightarrow 0$. Similarly, letting $t \rightarrow \infty$ and appealing to the beta-gamma algebra shows that $g_{\infty}$ under $\mathbb{P}_{0}^{\delta, \mu}$ is a $\operatorname{Gamma}\left(\alpha, \frac{1}{2} \mu^{2}\right)$ random variable; see also [14, Section 7].

Theorem 2.3 has a dual formulation in terms of the first zero after a fixed time which we state below as Theorem 2.6. We prove this directly and also show in Section 3.1 that either Theorem 2.3 or Theorem 2.6 can be deduced from the other using the time inversion property.

Theorem 2.6. Let $d_{t}=\inf \left\{s \geq t: X_{s}=0\right\}$ be the first zero after time $t$ of a Bessel process with dimension $\delta \in(0,2)$ and no drift starting at $x>0$. Put $\alpha=1-\frac{\delta}{2}$ and let 
$A_{\alpha}$ and $E_{x}$ be independent $\operatorname{Beta}(\alpha, 1-\alpha)$ and $\operatorname{Exp}\left(\frac{1}{2} x^{2}\right)$ random variables, respectively. Then we have the independent factorization

$$
d_{t} \text { under } \mathbb{P}_{x}^{\delta} \stackrel{\mathcal{L}}{=} \max \left\{t, \frac{1}{E_{x}}\right\} \frac{1}{A_{\alpha}} .
$$

Remark 2.7. By letting either $x \rightarrow 0$ or $t \rightarrow 0$, we see that $d_{t}$ under $\mathbb{P}_{0}^{\delta}$ is a shifted and scaled beta prime random variable while $\tau_{0}$ under $\mathbb{P}_{x}^{\delta}$ is an inverse gamma random variable.

\section{Preliminary results}

\subsection{Time inversion property and duality}

Our proofs rely on some well-known properties of Bessel processes and Bessel bridges which we now recall, starting with the aforementioned time inversion property [20, Theorem 2.1]. For $\delta>0$ and $x, \mu \geq 0$, this states that

$$
\left(t X_{\frac{1}{t}}: t>0 ; \mathbb{P}_{x}^{\delta, \mu}\right) \stackrel{\mathcal{L}}{=}\left(X_{t}: t>0 ; \mathbb{P}_{\mu}^{\delta, x}\right) .
$$

In other words, time inversion preserves dimension but swaps the drift and starting position of Bessel processes with drift. Since Bessel processes without drift satisfy the usual Brownian scaling property, we get from (3.1) and (2.4) that for any $\delta, c>0$

$$
\left(c X_{\frac{t}{c^{2}}}: t \geq 0 ; \mathbb{P}_{0}^{\delta, \mu}\right) \stackrel{\mathcal{L}}{=}\left(X_{t}: t \geq 0 ; \mathbb{P}_{0}^{\delta, \frac{\mu}{c}}\right) .
$$

The time inversion property (3.1) can also be used to establish a duality relation between $g_{t}$ and $d_{t}$. More precisely, for any $t>0$ and $x \geq 0$ with $\delta \in(0,2)$ we have

$$
\begin{aligned}
d_{t} \text { under } \mathbb{P}_{x}^{\delta} & \stackrel{\mathcal{L}}{=} \inf \left\{s \geq t: s X_{\frac{1}{s}}=0\right\} \text { under } \mathbb{P}_{0}^{\delta, x} \\
& =\frac{1}{\sup \left\{s \leq \frac{1}{t}: X_{s}=0\right\}} \text { under } \mathbb{P}_{0}^{\delta, x} \\
& =\frac{1}{g_{\frac{1}{t}}} \text { under } \mathbb{P}_{0}^{\delta, x} .
\end{aligned}
$$

Using (3.3), it is easy to deduce Theorem 2.6 from Theorem 2.3 and vice versa.

\subsection{Bessel bridges with $\delta>0$}

Next we introduce the notation $\mathbb{P}_{x \rightarrow y}^{\delta, T}$ for the law of a Bessel process with dimension $\delta>0$ which starts at $x \geq 0$ and is conditioned to be at $y \geq 0$ at time $T>0$, that is, the law of a Bessel bridge with dimension $\delta>0$ from $x$ to $y$ of length $T$. While the appearance of an arrow in the notation for bridge laws should prevent mistaking the $T$ for drift, we also use $B$ for the coordinate process of a bridge instead of $X$ to further the distinction.

The following lemma is a consequence of the time inversion property (3.1) and shows how Bessel processes with drift and Bessel bridges are related to each other through a space-time transformation. See [14, Theorem 5.8] for a similar result.

Lemma 3.1. Suppose $\delta, T>0$ and $\mu \geq 0$. Then we have:

i. $\left(B_{t}: 0 \leq t<T ; \mathbb{P}_{0 \rightarrow \mu}^{\delta, T}\right) \stackrel{\mathcal{L}}{=}\left((T-t) X_{\frac{t}{T(T-t)}}: 0 \leq t<T ; \mathbb{P}_{0}^{\delta, \mu}\right)$,

ii. $\left(X_{t}: t \geq 0 ; \mathbb{P}_{0}^{\delta, \mu}\right) \stackrel{\mathcal{L}}{=}\left(\frac{1+T t}{T} B_{\frac{T^{2} t}{1+T t}}: t \geq 0 ; \mathbb{P}_{0 \rightarrow \mu}^{\delta, T}\right)$. 
Proof. Starting from the definition of Bessel bridge, we can apply time inversion and then the Markov property to write

$$
\begin{aligned}
\left(B_{t}: 0<t \leq T ; \mathbb{P}_{0 \rightarrow \mu}^{\delta, T}\right) & \stackrel{\mathcal{L}}{=}\left(X_{t}: 0<t \leq T ; \mathbb{P}_{0}^{\delta} \mid X_{T}=\mu\right) \\
& \stackrel{\mathcal{L}}{=}\left(t X_{\frac{1}{t}}: 0<t \leq T ; \mathbb{P}_{0}^{\delta} \mid X_{\frac{1}{T}}=\frac{\mu}{T}\right) \\
& \stackrel{\mathcal{L}}{=}\left(t X_{\frac{1}{t}-\frac{1}{T}}: 0<t \leq T ; \mathbb{P}_{\frac{\mu}{T}}^{\delta}\right) .
\end{aligned}
$$

Applying time inversion again to the right-hand side of (3.4) and then using the scaling relation (3.2) results in

$$
\begin{aligned}
\left(t X_{\frac{1}{t}-\frac{1}{T}}: 0<t<T ; \mathbb{P}_{\frac{\mu}{T}}^{\delta}\right) & \stackrel{\mathcal{L}}{=}\left(\frac{T-t}{T} X_{\frac{T t}{T-t}}: 0<t<T ; \mathbb{P}_{0}^{\delta, \frac{\mu}{T}}\right) \\
& \stackrel{\mathcal{L}}{=}\left((T-t) X_{\frac{t}{T(T-t)}}: 0<t<T ; \mathbb{P}_{0}^{\delta, \mu}\right) .
\end{aligned}
$$

Now part i. follows from combining (3.4) with (3.5).

Part ii. can be deduced from part i. via

$$
\begin{aligned}
& \left(\frac{1+T t}{T} B_{\frac{T^{2} t}{1+T t}}: t \geq 0 ; \mathbb{P}_{0 \rightarrow \mu}^{\delta, T}\right) \\
& \stackrel{\mathcal{L}}{=}\left(\frac{1+T t}{T}\left(T-\frac{T^{2} t}{1+T t}\right) X \frac{\frac{T^{2} t}{1+T t}}{T\left(T-\frac{T^{2} t}{1+T t}\right)}: t \geq 0 ; \mathbb{P}_{0}^{\delta, \mu}\right) \\
& =\left(X_{t}: t \geq 0 ; \mathbb{P}_{0}^{\delta, \mu}\right) .
\end{aligned}
$$

\subsection{Bessel bridges with $\delta=0$}

It is well known that 0 is an absorbing state for the Bessel process of dimension 0 and this will have implications for the corresponding bridges. Before defining Bessel bridges in this case, we first recall the particularly simple distribution function of the absorption time. More generally, we let $\tau_{y}=\inf \left\{t>0: X_{t}=y\right\}$ denote the first hitting time of $y \in \mathbb{R}$ by the coordinate process. Then from [17, Corollary XI.1.4] we have

$$
\mathbb{P}_{x}^{0}\left(\tau_{0} \leq t\right)=e^{-\frac{x^{2}}{2 t}}, t>0
$$

From this it follows that

$$
\tau_{0} \text { under } \mathbb{P}_{x}^{0} \stackrel{\mathcal{L}}{=} \frac{1}{E_{x}}
$$

where $E_{x}$ is an $\operatorname{Exp}\left(\frac{1}{2} x^{2}\right)$ random variable.

Now we expound on the subtlety in the definition of Bessel bridges of dimension 0 that stems from 0 being an absorbing state for the underlying unconditioned process; see also [15, Section 5.3]. When $x>0$, the bridge law $\mathbb{P}_{x \rightarrow 0}^{0, T}$ results from conditioning a 0 -dimensional Bessel path of duration $T$ starting at $x$ to be absorbed before time $T$. Note that this conditioned absorption time will almost surely occur strictly between 0 and $T$. The bridge law $\mathbb{P}_{0 \rightarrow x}^{0, T}$ is simply the law of the time reversed bridge under $\mathbb{P}_{x \rightarrow 0}^{0, T}$, namely

$$
\left(B_{t}: 0 \leq t \leq T ; \mathbb{P}_{0 \rightarrow x}^{0, T}\right) \stackrel{\mathcal{L}}{=}\left(B_{T-t}: 0 \leq t \leq T ; \mathbb{P}_{x \rightarrow 0}^{0, T}\right) .
$$

When both $x, y>0$, the bridge law $\mathbb{P}_{x \rightarrow y}^{0, T}$ is defined just as in the $\delta>0$ case. When $x=y=0$, the law $\mathbb{P}_{0 \rightarrow 0}^{0, T}$ is degenerate and assigns probability 1 to the constant 0 path. 
By also conditioning on the exact time of absorption, we can relate a Bessel bridge of dimension 0 with a bridge of dimension 4 . More precisely, if $x>0$ and $0<S \leq T$, then we have

$$
\left(B_{t}: 0 \leq t \leq S ; \mathbb{P}_{x \rightarrow 0}^{0, T} \mid \tau_{0}=S\right) \stackrel{\mathcal{L}}{=}\left(B_{t}: 0 \leq t \leq S ; \mathbb{P}_{x \rightarrow 0}^{4, S}\right) .
$$

From this it follows by time reversal that

$$
\left(B_{t+T-S}: 0 \leq t \leq S ; \mathbb{P}_{0 \rightarrow x}^{0, T} \mid g_{T}=T-S\right) \stackrel{\mathcal{L}}{=}\left(B_{t}: 0 \leq t \leq S ; \mathbb{P}_{0 \rightarrow x}^{4, S}\right) .
$$

Lemma 3.1 does not apply when $\delta=0$ so we need another result for this case. The following lemma serves this purpose by using the same space-time transformation to connect a Bessel bridge of dimension 0 with the waiting Bessel process of dimension 4 which appears in Theorem 2.1.

Lemma 3.2. For $\mu>0$, let $E_{\mu}$ be an independent $\operatorname{Exp}\left(\frac{1}{2} \mu^{2}\right)$ random variable. Then

$$
\left((1+t) B_{\frac{t}{1+t}}: t \geq 0 ; \mathbb{P}_{0 \rightarrow \mu}^{0,1}\right) \stackrel{\mathcal{L}}{=}\left(X_{\left(t-E_{\mu}\right)^{+}}: t \geq 0 ; \mathbb{P}_{0}^{4, \mu}\right) .
$$

Proof. It follows from (3.8) that the bridge $\left(B_{t}: 0 \leq t \leq 1\right)$ under $\mathbb{P}_{0 \rightarrow \mu}^{0,1}$ can be split into two independent pieces by conditioning on $g_{1}$. Indeed, we can sample this bridge by first drawing $g_{1}$ under $\mathbb{P}_{0 \rightarrow \mu}^{0,1}$ and then sampling a bridge under $\mathbb{P}_{0 \rightarrow \mu}^{4,1-g_{1}}$ that is appended to a constant 0 path of length $g_{1}$. More precisely, let $\gamma$ be an independent random variable distributed like $g_{1}$ under $\mathbb{P}_{0 \rightarrow \mu}^{0,1}$. Then

$$
\left(B_{t}: 0 \leq t \leq 1 ; \mathbb{P}_{0 \rightarrow \mu}^{0,1}\right) \stackrel{\mathcal{L}}{=}\left(B_{(t-\gamma)^{+}}: 0 \leq t \leq 1 ; \mathbb{P}_{0 \rightarrow \mu}^{4,1-\gamma}\right) .
$$

Applying the space-time transformation to both sides of this equality in law results in

$$
\left((1+t) B_{\frac{t}{1+t}}: t \geq 0 ; \mathbb{P}_{0 \rightarrow \mu}^{0,1}\right) \stackrel{\mathcal{L}}{=}\left((1+t) B_{\left(\frac{t}{1+t}-\gamma\right)^{+}}: t \geq 0 ; \mathbb{P}_{0 \rightarrow \mu}^{4,1-\gamma}\right) .
$$

Since $0<\gamma<1$ almost surely, notice that

$$
\begin{aligned}
\left(\frac{t}{1+t}-\gamma\right)^{+} & = \begin{cases}0 & 0 \leq t<\frac{\gamma}{1-\gamma} \\
\frac{t}{1+t}-\gamma & t \geq \frac{\gamma}{1-\gamma}\end{cases} \\
& = \begin{cases}0 & 0 \leq t<\frac{\gamma}{1-\gamma} \\
\frac{(1-\gamma)(t-\gamma-\gamma t)}{(1-\gamma)(1+t)} & t \geq \frac{\gamma}{1-\gamma}\end{cases} \\
& =\frac{(1-\gamma)^{2}\left(t-\frac{\gamma}{1-\gamma}\right)^{+}}{1+(1-\gamma)\left(t-\frac{\gamma}{1-\gamma}\right)^{+}}
\end{aligned}
$$

and similar calculations show that

$$
1+t=\frac{1+(1-\gamma)\left(t-\frac{\gamma}{1-\gamma}\right)^{+}}{1-\gamma}, t \geq \frac{\gamma}{1-\gamma} .
$$

Writing $f(t)$ for the $\left(t-\frac{\gamma}{1-\gamma}\right)^{+}$which appears in both of these identities, now we can make the appropriate substitutions in the right-hand side of (3.9) and then appeal to part ii. of Lemma 3.1 to yield

$$
\begin{aligned}
& \left((1+t) B_{\frac{t}{1+t}}: t \geq 0 ; \mathbb{P}_{0 \rightarrow \mu}^{0,1}\right) \\
& \stackrel{\mathcal{E}}{=}\left(\left(\frac{1+(1-\gamma) f(t)}{1-\gamma}\right) B_{\frac{(1-\gamma)^{2} f(t)}{1+(1-\gamma) f(t)}}: t \geq 0 ; \mathbb{P}_{0 \rightarrow \mu}^{4,1-\gamma}\right) \\
& \underline{\underline{\mathcal{L}}}\left(X_{\left(t-\frac{\gamma}{1-\gamma}\right)^{+}}: t \geq 0 ; \mathbb{P}_{0}^{4, \mu}\right) .
\end{aligned}
$$


It remains to identify the distribution of the $\frac{\gamma}{1-\gamma}$ appearing in (3.10). By hypothesis, this random variable is distributed like $\frac{g_{1}}{1-g_{1}}$ under $\mathbb{P}_{0 \rightarrow \mu}^{0,1}$ and is also independent of the Bessel process that appears in (3.10). From (3.6), it follows that $g_{1}$ under $\mathbb{P}_{0 \rightarrow \mu}^{0,1}$ has distribution function

$$
\begin{aligned}
\mathbb{P}_{0 \rightarrow \mu}^{0,1}\left(g_{1} \leq t\right) & =\mathbb{P}_{\mu \rightarrow 0}^{0,1}\left(1-\tau_{0} \leq t\right) \\
& =1-e^{-\frac{\mu^{2} t}{2(1-t)}}
\end{aligned}
$$

Hence

$$
\begin{aligned}
\mathbb{P}_{0 \rightarrow \mu}^{0,1}\left(\frac{g_{1}}{1-g_{1}} \leq t\right) & =\mathbb{P}_{0 \rightarrow \mu}^{0,1}\left(g_{1} \leq \frac{t}{1+t}\right) \\
& =1-e^{-\frac{1}{2} \mu^{2} t} .
\end{aligned}
$$

From this we deduce that the $\frac{\gamma}{1-\gamma}$ appearing in (3.10) is an independent $\operatorname{Exp}\left(\frac{1}{2} \mu^{2}\right)$ random variable and the proof is complete.

\subsection{Additivity property}

Lastly, we recall the additivity property of squared Bessel processes [17, Theorem XI.1.2]. This property states that if $X$ and $X^{\prime}$ are independent squared Bessel processes of dimensions $\delta, \delta^{\prime} \geq 0$ starting from $x, x^{\prime} \geq 0$, then their sum $\left(X_{t}+X_{t}^{\prime}: t \geq 0\right)$ is a squared Bessel process of dimension $\delta+\delta^{\prime}$ starting from $x+x^{\prime}$. A more succinct statement of the additivity property is

$$
\mathbb{Q}_{x}^{\delta} * \mathbb{Q}_{x^{\prime}}^{\delta^{\prime}}=\mathbb{Q}_{x+x^{\prime}}^{\delta+\delta^{\prime}}
$$

where we used $\mathbb{Q}_{x}^{\delta} * \mathbb{Q}_{x^{\prime}}^{\delta^{\prime}}$ to denote the law of the sum of independent processes with laws $\mathbb{Q}_{x}^{\delta}$ and $\mathbb{Q}_{x^{\prime}}^{\delta^{\prime}}$. The additivity property also applies to squared Bessel bridges and for $\delta, \delta^{\prime} \geq 0$ and $x, x^{\prime} \geq 0$ we have the statements

$$
\begin{gathered}
\mathbb{Q}_{x \rightarrow 0}^{\delta, T} * \mathbb{Q}_{x^{\prime} \rightarrow 0}^{\delta^{\prime}, T}=\mathbb{Q}_{x+x^{\prime} \rightarrow 0}^{\delta+\delta^{\prime}, T} \\
\mathbb{Q}_{0 \rightarrow x}^{\delta, T} * \mathbb{Q}_{0 \rightarrow x^{\prime}}^{\delta^{\prime}, T}=\mathbb{Q}_{0 \rightarrow x+x^{\prime}}^{\delta+\delta^{\prime}, T} .
\end{gathered}
$$

The analogous result for bridges with general starting and ending points is more complicated; see [15, Theorem 5.8].

\section{Proofs of the main theorems}

\subsection{Proof of Theorem 2.1}

Proof of Theorem 2.1. We apply part ii. of Lemma 3.1, the additivity property for bridges (3.12), then part ii. of Lemma 3.1 and Lemma 3.2 to write

$$
\begin{aligned}
& \left(X_{t}^{2}: t \geq 0 ; \mathbb{P}_{0}^{\delta, \mu}\right) \\
& \stackrel{\mathcal{L}}{=}\left((1+t)^{2} B_{\frac{t}{1+t}}^{2}: t \geq 0 ; \mathbb{P}_{0 \rightarrow \mu}^{\delta, 1}\right) \\
& \stackrel{\mathcal{L}}{=}\left(\left((1+t) B_{\frac{t}{1+t}}\right)^{2}+\left((1+t) B_{\frac{t}{1+t}}^{\prime}\right)^{2}: t \geq 0 ; \mathbb{P}_{0 \rightarrow 0}^{\delta, 1}(B) \times \mathbb{P}_{0 \rightarrow \mu}^{0,1}\left(B^{\prime}\right)\right) \\
& \stackrel{\mathcal{L}}{=}\left(X_{t}^{2}+X_{\left(t-E_{\mu}\right)^{+}}^{\prime 2}: t \geq 0 ; \mathbb{P}_{0}^{\delta}(X) \times \mathbb{P}_{0}^{4, \mu}\left(X^{\prime}\right)\right) .
\end{aligned}
$$

In light of (2.2), this proves the theorem. 


\subsection{Two proofs of Theorem 2.3}

Our first proof of Theorem 2.3 uses a formula found on page 27 of Borodin and Salminen's handbook [2] that holds for regular diffusions. More specifically, let $p_{t}^{\delta, \mu}(x, y)$ denote the transition density with respect to the speed measure of the Bessel process with dimension $\delta$ and drift $\mu$ and let $G_{\lambda}^{\delta, \mu}(x, y):=\int_{0}^{\infty} e^{-\lambda t} p_{t}^{\delta, \mu}(x, y) \mathrm{d} t$ be the corresponding Green function. Keeping with our previous notation for the law of Bessel processes with and without drift, we omit $\mu$ when it is equal to 0 . Furthermore, let $f^{\delta, \mu}$ denote the inverse Laplace transform of $\left(\lambda G_{\lambda}^{\delta, \mu}(0,0)\right)^{-1}$ with respect to $\lambda$, that is, $f^{\delta, \mu}$ satisfies

$$
\begin{aligned}
\mathcal{L}\left\{f^{\delta, \mu}\right\}(\lambda): & =\int_{0}^{\infty} e^{-\lambda s} f^{\delta, \mu}(s) \mathrm{d} s \\
& =\frac{1}{\lambda G_{\lambda}^{\delta, \mu}(0,0)}, \lambda>0 .
\end{aligned}
$$

It will be convenient to write this relation in terms of the inverse Laplace transform as

$$
\mathcal{L}^{-1}\left\{\frac{1}{\lambda G_{\lambda}^{\delta, \mu}(0,0)}\right\}(s)=f^{\delta, \mu}(s), s>0 .
$$

Now we can state the formula from the handbook [2] which asserts that

$$
\mathbb{P}_{0}^{\delta, \mu}\left(g_{t} \in \mathrm{d} s\right)=f^{\delta, \mu}(t-s) p_{s}^{\delta, \mu}(0,0) \mathrm{d} s, 0<s<t .
$$

Proof 1 of Theorem 2.3. It follows from the absolute continuity relation (2.3) that

$$
G_{\lambda}^{\delta, \mu}(0,0)=G_{\lambda+\frac{1}{2} \mu^{2}}^{\delta}(0,0) .
$$

Hence we can use properties of the Laplace transform and its inverse to write

$$
\begin{aligned}
f^{\delta, \mu}(s) & =\mathcal{L}^{-1}\left\{\frac{\lambda+\frac{1}{2} \mu^{2}}{\lambda} \frac{1}{\left(\lambda+\frac{1}{2} \mu^{2}\right) G_{\lambda+\frac{1}{2} \mu^{2}}^{\delta}(0,0)}\right\}(s) \\
& =\int_{0}^{s} \mathcal{L}^{-1}\left\{\frac{\lambda+\frac{1}{2} \mu^{2}}{\lambda}\right\}(s-u) e^{-\frac{1}{2} \mu^{2} u} \mathcal{L}^{-1}\left\{\frac{1}{\lambda G_{\lambda}^{\delta}(0,0)}\right\}(u) \mathrm{d} u \\
& =\int_{0}^{s}\left(\delta_{0}(s-u)+\frac{1}{2} \mu^{2}\right) e^{-\frac{1}{2} \mu^{2} u} f^{\delta}(u) \mathrm{d} u \\
& =e^{-\frac{1}{2} \mu^{2} s} f^{\delta}(s)+\int_{0}^{s} \frac{1}{2} \mu^{2} e^{-\frac{1}{2} \mu^{2} u} f^{\delta}(u) \mathrm{d} u, s>0 .
\end{aligned}
$$

Here we used $\delta_{0}(\cdot)$ for the Dirac delta. Using (4.2) in (4.1) along with the absolute continuity relation (2.3) leads to the desired density on the interval $(0, t)$ of $g_{t}$ under $\mathbb{P}_{0}^{\delta, \mu}$

$$
\mathbb{P}_{0}^{\delta, \mu}\left(g_{t} \in \mathrm{d} s\right)=\left(e^{-\frac{1}{2} \mu^{2} t} f^{\delta}(t-s)+\int_{0}^{t-s} \frac{1}{2} \mu^{2} e^{-\frac{1}{2} \mu^{2}(u+s)} f^{\delta}(u) \mathrm{d} u\right) p_{s}^{\delta}(0,0) \mathrm{d} s .
$$

Next we compute the density of the right-hand side of (2.5) and show that it is equal to (4.3). We condition on $E_{\mu}$ and use Lamperti's arcsine law (1.2) and then (4.1) to write

$$
\begin{aligned}
P & \left(\min \left\{t, E_{\mu}\right\} A_{\alpha} \leq s\right) \\
& =e^{-\frac{1}{2} \mu^{2} t} P\left(t A_{\alpha} \leq s\right)+\int_{0}^{t} \frac{1}{2} \mu^{2} e^{-\frac{1}{2} \mu^{2} u} P\left(u A_{\alpha} \leq s\right) \mathrm{d} u \\
& =e^{-\frac{1}{2} \mu^{2} t} \mathbb{P}_{0}^{\delta}\left(g_{t} \leq s\right)+\int_{0}^{t} \frac{1}{2} \mu^{2} e^{-\frac{1}{2} \mu^{2} u} \mathbb{P}_{0}^{\delta}\left(g_{u} \leq s\right) \mathrm{d} u \\
& =e^{-\frac{1}{2} \mu^{2} t} \int_{0}^{s} f^{\delta}(t-w) p_{w}^{\delta}(0,0) \mathrm{d} w+\int_{0}^{t} \frac{1}{2} \mu^{2} e^{-\frac{1}{2} \mu^{2} u} \int_{0}^{s \wedge u} f^{\delta}(u-w) p_{w}^{\delta}(0,0) \mathrm{d} w \mathrm{~d} u \\
& =e^{-\frac{1}{2} \mu^{2} t} \int_{0}^{s} f^{\delta}(t-w) p_{w}^{\delta}(0,0) \mathrm{d} w+\int_{0}^{s} \int_{w}^{t} \frac{1}{2} \mu^{2} e^{-\frac{1}{2} \mu^{2} u} f^{\delta}(u-w) p_{w}^{\delta}(0,0) \mathrm{d} u \mathrm{~d} w .
\end{aligned}
$$


Now differentiating (4.4) with respect to $s$ results in

$$
\begin{aligned}
\frac{\mathrm{d}}{\mathrm{d} s} P\left(\min \left\{t, E_{\mu}\right\} A_{\alpha} \leq s\right) & =\left(e^{-\frac{1}{2} \mu^{2} t} f^{\delta}(t-s)+\int_{s}^{t} \frac{1}{2} \mu^{2} e^{-\frac{1}{2} \mu^{2} u} f^{\delta}(u-s) \mathrm{d} u\right) p_{s}^{\delta}(0,0) \\
& =\left(e^{-\frac{1}{2} \mu^{2} t} f^{\delta}(t-s)+\int_{0}^{t-s} \frac{1}{2} \mu^{2} e^{-\frac{1}{2} \mu^{2}(u+s)} f^{\delta}(u) \mathrm{d} u\right) p_{s}^{\delta}(0,0) .
\end{aligned}
$$

Since this agrees with (4.3), the densities are equal and the theorem is proved.

Our second proof of Theorem 2.3 is more direct and also avoids the computations and Laplace transform machinery of the first proof. More importantly, the second proof provides a probabilistic explanation by showing how the independent factorization is a natural consequence of the additive decomposition from Theorem 2.1.

Proof 2 of Theorem 2.3. Since $X^{\prime}$ under $\mathbb{Q}_{0}^{4, \mu}$ never returns to 0 once it starts, we can use the additive decomposition from Theorem 2.1 to write

$$
\begin{aligned}
& \sup \left\{s \leq t: X_{s}=0\right\} \text { under } \mathbb{Q}_{0}^{\delta, \mu} \\
& \quad \mathcal{L} \sup \left\{s \leq t: X_{s}+X_{\left(s-E_{\mu}\right)^{+}}^{\prime}=0\right\} \text { under } \mathbb{Q}_{0}^{\delta}(X) \times \mathbb{Q}_{0}^{4, \mu}\left(X^{\prime}\right) \\
& \stackrel{\mathcal{L}}{=} \sup \left\{s \leq \min \left\{t, E_{\mu}\right\}: X_{s}=0\right\} \text { under } \mathbb{Q}_{0}^{\delta} .
\end{aligned}
$$

We can use the independence of $E_{\mu}$ and $X$ along with Bessel scaling to factor out the $\min \left\{t, E_{\mu}\right\}$ from inside the sup appearing in (4.5). Together with the fact that the zeros of a process and its square are the same, this allows us to conclude that

$$
\begin{aligned}
g_{t} \text { under } \mathbb{P}_{0}^{\delta, \mu} & \stackrel{\mathcal{L}}{=} \min \left\{t, E_{\mu}\right\} \sup \left\{s \leq 1: X_{s}=0\right\} \text { under } \mathbb{Q}_{0}^{\delta} \\
& \stackrel{\mathcal{L}}{=} \min \left\{t, E_{\mu}\right\} g_{1} \text { under } \mathbb{P}_{0}^{\delta} .
\end{aligned}
$$

Now the desired result follows from Lamperti's arcsine law (1.2).

\subsection{Proof of Theorem 2.6}

As mentioned in Section 3.1, Theorem 2.6 follows from a combination of Theorem 2.3 and the duality relation (3.3). Here we opt for a direct proof based on the well-known additivity property for squared Bessel processes without drift discussed in Section 3.4.

Proof of Theorem 2.6. Since 0 is an absorbing state for $X^{\prime}$ under $\mathbb{Q}_{x^{2}}^{0}$, we can use the additivity property (3.11) to write

$$
\begin{aligned}
& \inf \left\{s \geq t: X_{s}=0\right\} \text { under } \mathbb{Q}_{x^{2}}^{\delta} \\
& \quad \stackrel{\mathcal{L}}{=} \inf \left\{s \geq t: X_{s}+X_{s}^{\prime}=0\right\} \text { under } \mathbb{Q}_{0}^{\delta}(X) \times \mathbb{Q}_{x^{2}}^{0}\left(X^{\prime}\right) \\
& \stackrel{\mathcal{L}}{=} \inf \left\{s \geq \max \left\{t, \tau_{0}^{\prime}\right\}: X_{s}=0\right\} \text { under } \mathbb{Q}_{0}^{\delta}(X) \times \mathbb{Q}_{x^{2}}^{0}\left(X^{\prime}\right) .
\end{aligned}
$$

The independence of $\tau_{0}^{\prime}$ and $X$ allows us to apply Bessel scaling to (4.6), thereby factoring out the $\max \left\{t, \tau_{0}^{\prime}\right\}$ from inside the inf. In conjunction with (2.2) and (3.7), this implies that

$$
\begin{aligned}
d_{t} \text { under } \mathbb{P}_{x}^{\delta} & \stackrel{\mathcal{L}}{=} \max \left\{t, \tau_{0}^{\prime}\right\} \inf \left\{s \geq 1: X_{s}=0\right\} \text { under } \mathbb{Q}_{0}^{\delta}(X) \times \mathbb{Q}_{x^{2}}^{0}\left(X^{\prime}\right) \\
& \stackrel{\mathcal{L}}{=} \max \left\{t, \frac{1}{E_{x}}\right\} d_{1} \text { under } \mathbb{P}_{0}^{\delta} .
\end{aligned}
$$

Now we can use (3.3) and (1.2) to rewrite (4.7) as

$$
d_{t} \text { under } \mathbb{P}_{x}^{\delta} \stackrel{\mathcal{L}}{=} \max \left\{t, \frac{1}{E_{x}}\right\} \frac{1}{A_{\alpha}}
$$

which completes the proof. 
Last zero arcsine law for Bessel processes with drift

\section{References}

[1] L. Alili, P. Graczyk, and T. Żak, On inversions and Doob h-transforms of linear diffusions, In Memoriam Marc Yor - Séminaire de Probabilités XLVII, Lecture Notes in Mathematics, vol. 2137, Springer, 2015, pp. 107-126. MR-3444296

[2] A. N. Borodin and P. Salminen, Handbook of Brownian Motion - Facts and Formulae, second ed., Probability and its Applications, Birkhäuser Verlag, Basel, 2002. MR-1912205

[3] esg (https://mathoverflow.net/users/48831/esg), Arcsine law for Brownian motion with drift, URL (version: 2016-06-09): https://mathoverflow.net/q/223594.

[4] F. Iafrate and E. Orsingher, The last zero-crossing of an iterated brownian motion with drift, Stochastics 92 (2020), no. 3, 356-378. MR-4085756

[5] J. Jakubowski and M. Wiśniewolski, On hyperbolic Bessel processes and beyond, Bernoulli 19 (2013), no. 5B, 2437-2454. MR-3160560

[6] J. Lamperti, An invariance principle in renewal theory, Ann. Math. Statist. 33 (1962), 685-696. MR-137176

[7] S. Lawi, Towards a characterization of Markov processes enjoying the time-inversion property, J. Theoret. Probab. 21 (2008), no. 1, 144-168. MR-2384476

[8] P. Lévy, Sur certains processus stochastiques homogènes, Compositio Math. 7 (1939), 283339. MR-919

[9] V. Linetsky, The spectral representation of Bessel processes with constant drift: applications in queueing and finance, J. Appl. Probab. 41 (2004), no. 2, 327-344. MR-2052575

[10] R. Mansuy and M. Yor, Aspects of Brownian motion, Universitext, Springer-Verlag, Berlin, 2008. MR-2454984

[11] R. G. Pinsky, Positive harmonic functions and diffusion, Cambridge Studies in Advanced Mathematics, vol. 45, Cambridge University Press, Cambridge, 1995. MR-1326606

[12] J. Pitman, Random weighted averages, partition structures and generalized arcsine laws, arXiv:1804.07896, 2018.

[13] J. Pitman and M. Winkel, Squared Bessel processes of positive and negative dimension embedded in Brownian local times, Electron. Commun. Probab. 23 (2018), paper no. 74, 13 pp. MR-3866047

[14] J. Pitman and M. Yor, Bessel processes and infinitely divisible laws, Stochastic Integrals, Lecture Notes in Mathematics, vol. 851, Springer, Berlin, 1981, pp. 285-370. MR-620995

[15] J. Pitman and M. Yor, A decomposition of Bessel bridges, Z. Wahrsch. Verw. Gebiete 59 (1982), no. 4, 425-457. MR-656509

[16] A. Pyć, G. Serafin, and T. Żak, Supremum distribution of Bessel process of drifting Brownian motion, Probab. Math. Statist. 35 (2015), no. 2, 201-222. MR-3433649

[17] D. Revuz and M. Yor, Continuous martingales and Brownian motion, third ed., Grundlehren der Mathematischen Wissenschaften, vol. 293, Springer-Verlag, Berlin, 1999. MR-1725357

[18] L. C. G. Rogers and J. W. Pitman, Markov functions, Ann. Probab. 9 (1981), no. 4, 573-582. MR-624684

[19] E. Schulte-Geers and W. Stadje, Small drift limit theorems for random walks, J. Appl. Probab. 54 (2017), no. 1, 199-212. MR-3632614

[20] S. Watanabe, On time inversion of one-dimensional diffusion processes, Z. Wahrscheinlichkeitstheorie und Verw. Gebiete 31 (1975), 115-124. MR-365731

[21] D. Williams, Path decomposition and continuity of local time for one-dimensional diffusions I, Proc. London Math. Soc. 28 (1974), no. 4, 738-768. MR-350881

[22] M. Yor, On square-root boundaries for Bessel processes, and pole-seeking Brownian motion, Stochastic Analysis and Applications, Lecture Notes in Mathematics, vol. 1095, Springer, Berlin, 1984, pp. 100-107. MR-777516

Acknowledgments. The author would like to thank Jim Pitman and Ernst Schulte-Geers for providing useful comments on earlier drafts and also thank an anonymous referee for pointing out formula (4.1) from Borodin and Salminen's handbook [2]. 


\section{Electronic Journal of Probability Electronic Communications in Probability}

\section{Advantages of publishing in EJP-ECP}

- Very high standards

- Free for authors, free for readers

- Quick publication (no backlog)

- Secure publication $\left(\mathrm{LOCKSS}^{1}\right)$

- Easy interface (EJMS²)

\section{Economical model of EJP-ECP}

- Non profit, sponsored by $\mathrm{IMS}^{3}, \mathrm{BS}^{4}$, ProjectEuclid ${ }^{5}$

- Purely electronic

\section{Help keep the journal free and vigorous}

- Donate to the IMS open access fund ${ }^{6}$ (click here to donate!)

- Submit your best articles to EJP-ECP

- Choose EJP-ECP over for-profit journals

\footnotetext{
${ }^{1}$ LOCKSS: Lots of Copies Keep Stuff Safe http://www. lockss.org/

${ }^{2}$ EJMS: Electronic Journal Management System http://www.vtex.lt/en/ejms.html

${ }^{3}$ IMS: Institute of Mathematical Statistics http://www.imstat.org/

${ }^{4}$ BS: Bernoulli Society http://www. bernoulli-society.org/

${ }^{5}$ Project Euclid: https://projecteuclid.org/

${ }^{6}$ IMS Open Access Fund: http://www.imstat.org/publications/open.htm
} 